\title{
A relação entre a ferramenta de Construção de Cenários Futuros e as estratégias metacognitivas: uma abordagem das Neurociências acerca do Design
}

The relationship between the Design Orienting Scenarios tool and metacognitive strategies: a Neuroscience approach to Design

VANZ, Samanta; Mestranda; Universidade de Caxias do Sul - UCS.

SVanz1@ucs.br

\section{Resumo}

O presente trabalho relaciona as estratégias metacognitivas com a ferramenta de construção de cenários futuros, utilizada de forma coletiva para auxiliar a tomada de decisão dentro do contexto projetual. Para a operacionalização dos objetivos, foram elaborados três instrumentos para a avaliação dos dados: as fichas de estrutura, que procuraram descrever a estrutura da ferramenta; o formulário, para descrever a composição estrutural da ferramenta e as relações entre as fases; e o inventário de análise, que procurou identificar elementos da metacognição. Os dados sugeriram que a ferramenta de construção de cenários futuros permite explorar em maior grau as estratégias metacognitivas relacionadas ao planejamento e ao gerenciamento de informação, sendo possível presumir que a mesma é um instrumento importante para a aprendizagem colaborativa em contextos de projetos, e a introdução de elementos da metacognição em sua estrutura pode potencializar o uso desta ferramenta para a aprendizagem.

Palavras Chave: ferramenta de construção de cenários futuros; metacognição; aprendizagem

\begin{abstract}
This study focused to relate the metacognitive strategies with the aspects present in Design Orienting Scenarios, a tool of scenario building technique, in teaching and learning groups, to assist decision making in design context. Three instruments for evaluation of the data were elaborated to achieve the purposes: structure cards, to decompose and describe its structure; the form, to describe its structural composition and the relationship between the phases; and the analysis inventory, to identify the elements of metacognition present in a case study. The analysis of the data suggested that Design Orienting Scenarios tool allows to explore the metacognitive strategies related to planning and information management. Then, it was possible to assume that the Design Orienting Scenarios tool is an important instrument for collaborative learning in project contexts, and the application of metacognitive strategies in its structure can enhance the use of this tool for learning in groups.
\end{abstract}

Keywords: Design Orienting Scenarios tool; metacognition; learning 


\section{Introdução}

A ferramenta de construção de cenários futuros procura auxiliar a tomada de decisões no contexto projetual, como uma forma de gerar a visão hipotética de uma situação futura através da análise dos dados atuais, contemplando o contexto externo de um projeto, os atores envolvidos neste contexto e a interação dos dados que relacionam estes dois pontos (MANZINI; JÉGOU; MERONI, 2004). Para Manzini, Jégou e Meroni (2004), a função dos cenários é atuar como uma plataforma de interação, possibilitando a articulação de propostas de projeto e criando material estratégico para o suporte de análises e avaliações.

Esta ferramenta procura trabalhar com a construção de um mapa de possibilidades, que viabiliza a tomada de decisões, facilita a aprendizagem organizacional, simula e avalia os impactos de ações futuras além de representar possíveis referências em termos de contexto, situações de uso, relações usuário-produto, significados e efeitos de sentido (MANZINI; JÉGOU; MERONI, 2004).

Refletir e avaliar as variáveis existentes, que necessitam de monitoramento e revisão a respeito do quê e o quanto se sabe sobre um contexto específico, são ações esperadas durante o processo de construção de cenários (MANZINI; JÉGOU, MERONI, 2004).

O uso de elementos de monitoramento e regulaçãosão características também identificadas nos processos metacognitivos (FLAVELL; MILLER; MILLER, 1999). A metacognição pode ser descrita como o conhecimento sobre o conhecimento, sendo geralmente expressada por meio de uma série de julgamentos que refletem o domínio do saber individual (FELTES, 2014). A metacognição influencia processos mentais como a percepção, a atenção, a memória, a solução de problemas, a cognição social e as formas de autocontrole e de autoinstrução, o que a torna uma ferramenta cognitiva de diversas aplicações (FLAVELL; MILLER; MILLER, 1999).

Partindo desta explanação, o presente trabalho levanta o seguinte problema: que aspectos da ferramenta de criação de cenários futuros podem ser relacionados com estratégias metacognitivas em contexto de ensino e aprendizagem que possua o projeto como atividade principal?

Ao pensar na possibilidade da relação entre metacognição e a ferramenta de criação de cenários futuros, é possível identificar pontos importantes dentro do processo de desenvolvimento desta ferramenta que podem ser amparados pelos conhecimentos metacognitivos: compreender a finalidade da atividade, planejar a sua realização, aplicar e alterar conscientemente estratégias de aplicação e avaliar o seu próprio processo de execução (RIBEIRO, 2003; MANZINI; JÉGOU; MERONI, 2004).

Por se tratar de um conhecimento que, a grosso modo, refere-se ao gerenciamento do saber, considera-se que, empreendendo os conceitos da ferramenta de construção de cenários futuros juntamente com a metacognição, é possível adaptar esta ferramenta e torná-la passível de uso específico não apenas na aplicação de projetos, mas no contexto de construção do conhecimento sobre projetos em diferentes áreas, colaborando em campos como o design, a administração, a publicidade, que necessitam de conhecimentos específicos em ferramentas que facilitem e amparem a relação entre ensinar e aprender durante o processo de projetar.

É importante identificar que esta ferramenta pode colaborar também com o conhecimento de profissionais que trabalham em grupos, em muitas circunstâncias multidisciplinares, que necessitam ter um entendimento mútuo a respeito de um determinado problema, convergindo em 
interesses comuns e até mesmo possíveis sinergias para identificar uma solução dentro do limite a ser analisado (MANZINI; JÉGOU; MERONI, 2004). A construção do conhecimento pode ser facilitada ao se utilizar informações e estratégias que colaborem com o ato de aprender, sendo que a construção cognitiva de quem trabalha em grupos é também o resultado das interações que se desenvolvem através da relação entre os indivíduos (BEBER; SILVA; BONFIGLIO, 2014; FLAVELL; MILLER; MILLER, 1999).

\section{Ferramenta de Construção de Cenários Futuros e Metacognição}

O quadro teórico a ser analisado dentro da perspectiva do design é apresentado pelos autores Manzini, Jégou e Meroni, com o termo Design Orienting Scenarios. Esta é uma ferramenta em constante evolução, que tem como uma das principais características a atuação de diferentes atores dentro de um mesmo contexto projetual (MANZINI; JÉGOU; MERONI, 2004).

Como Manzini, Jégou e Meroni (2004) pontuam, o termo cenário é aplicado como o sinônimo de uma visão sistêmica a respeito de um conjunto de possibilidades, de alternativas, a respeito de algo que é articulado e complexo. O termo design orienting surge a partir da consideração de que esta visão traz um framework projetual para novos produtos e serviços.

A construção de cenários futuros pode ser considerada uma ferramenta que possibilita pensar diferentes aspectos de um contexto projetual através da combinação de tendências e políticas, facilitando a tomada de decisões, a flexibilidade da análise dos resultados e possibilita a inovação (AMER; DAIM; JETTER, 2013).

Dentro deste contexto, os cenários resultam de modelos mentais compartilhados e acordados entre um grupo específico de pessoas, os atores, expressos como descrições narrativas e diagramas visuais consistentes que procuram ilustrar futuros possíveis (VAN DER HEIJDEN, 2009; MANZINI; JÉGOU; MERONI, 2004).

Os cenários não podem ser considerados o único fator decisório para a continuidade de um projeto; é importante entender que os mesmos funcionam como um mecanismo para produzir as informações que são relevantes para as decisões projetuais, mas as decisões não podem ser baseadas a partir de um cenário ser mais provável que outro. Dentro da ferramenta de construção de cenários futuros, todos os cenários devem ser tratados com o mesmo peso, sendo todos plausíveis, com probabilidade de retornos positivos (VAN DER HEIJDEN, 2009).

Design Orienting Scenarios, ou como será chamado neste trabalho, "Construção de Cenários Futuros" (CCF), é estruturado a partir de três elementos fundamentais: a visão, que é uma imagem hipotética do futuro; a motivação, que são as intenções que justificam e dão significado ao cenário; e a proposta, que é a aplicação deste cenário, com seu sistema de produtos e serviços (MANZINI; JÉGOU; MERONI, 2004; HARTMANN; FRANZATO; SCALETSKY; REYES, 2012).

Por meio destes elementos fundamentais, a ferramenta de construção de cenários futuros pode ser dividida em fases onde ocorre o processo de coleta de informação, de discussão, da elaboração dos cenários e de sua representação gráfica, conforme apresentado na Figura 1 (MANZINI; JÉGOU; MERONI, 2004): 
Figura 1 - Esquema representacional da ferramenta de construção de cenários futuros

construção de cenários futuros

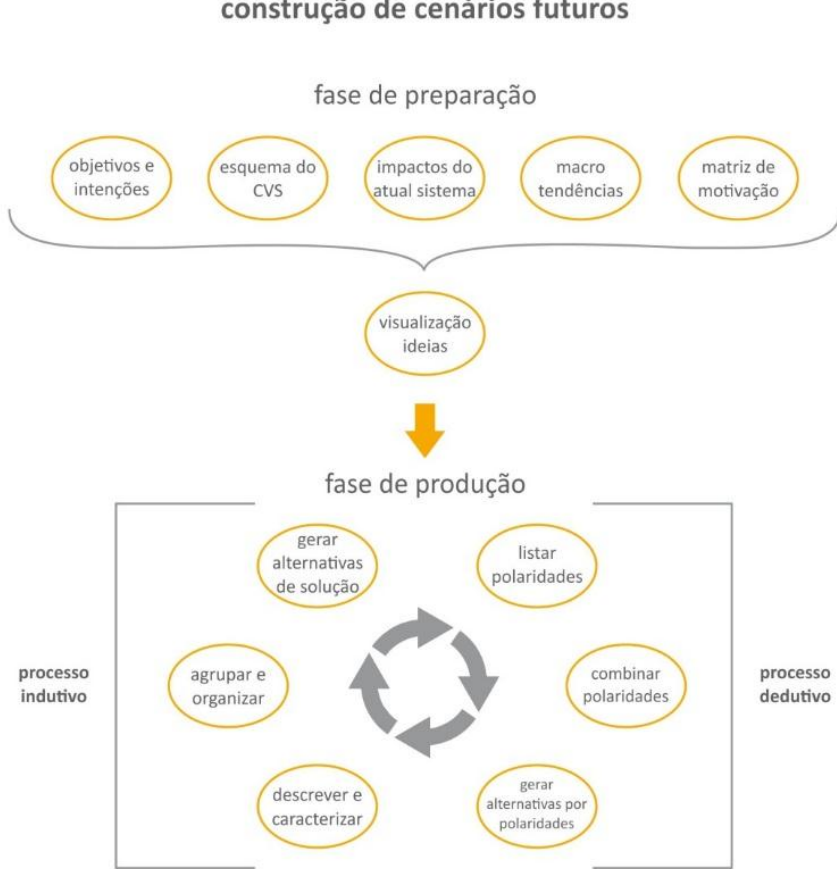

fase de construção dos cenários

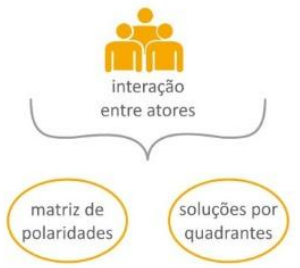

fase de representações
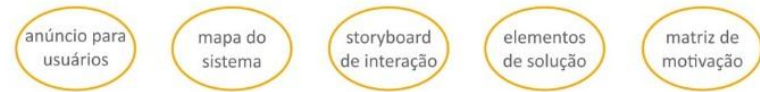

Existe, no decorrer do uso desta ferramenta, o objetivo de analisar o sistema atual ao qual o projeto está inserido, dialogar e definir os pontos comuns que se deseja alcançar por meio do projeto e finalmente gerar as ideias para solucionar o problema projetual com base na organização de informações diagramadas (MANZINI; JÉGOU, MERONI, 2004).

Além disso, a ferramenta CCF baseia-se na interação de dois processos complementares: indutivo e dedutivo. Na fase indutiva, ocorre um processo bottom-up, que é caracterizado pela organização das premissas dos sistemas atuais, sejam produtos ou serviços, com o objetivo de reconfigurá-los. Na fase dedutiva, ocorre um processo top-down, onde há a exploração da reconfiguração dos sistemas atuais, criando cenários alternativos para a solução dos problemas projetuais (MANZINI; JÉGOU; MERONI, 2004; JÉGOU; MANZINI, 2008; MERONI, 2007). 
Para que a ferramenta atinja o objetivo da geração de cenários futuros que facilitem a tomada de decisão é necessário que os atores envolvidos estejam atentos aos sinais fracos identificados durante a fase de preparação, sejam capazes de adaptar os dados coletados à fase de produção e projetem a integração da experiência, da significância dos dados e da ação em um repertório holístico (VAN DER HEIJDEN, 2009), podendo ser utilizada para a convergência de ideias e o compartilhamento de visões dentro de um contexto onde haja envolvimento de muitos atores.

Estas inferências que resultam da reflexão individual e da colaboração podem ser amparadas pelos conceitos explorados na metacognição, que é o domínio referente a diferenciar, analisar, monitorar, organizar, avaliare modificar os próprios processos cognitivos, is to é, o conhecimento que o indivíduo tem a respeito de seu próprio conhecimento e que o permite agir sobre este conhecimento (RIBEIRO, 2003; CATANIA; 1999). O processo de pensar como se pensa possibilita construir formas de autoconhecimento, identificar como funcionam nossos proces sos cognitivos: julgamentos, inferências, tomada de decisões (FELTES, 2014).

Para Flavell, Miller e Miller (1999, pág. 126), a metacognição refere-se ao "conhecimento metacognitivo e ao monitoramento e autorregulação metacognitivos", que são esferas do conhecimento relacionadas com a consciência de processos de uma determinada tarefa, quais as competências necessárias para concluí-la e o controle da atividade cognitiva durante a realização desta tarefa, o que permite realizar avaliações e correções no decorrer de sua execução (RIBEIRO, 2003).

O conhecimento metacognitivo (FLAVELL; MILLER; MILLER, 1999; RIBEIRO, 2003) diz respeito ao conhecimento e as crenças que o indivíduo tem a respeito de si próprio, aquilo que resulta das experiências e é armazenado na memória a longo prazo, e possui três diferentes subcategorias: a) conhecimento sobre pessoas; b) conhecimento sobre tarefas; c) conhecimento sobre estratégias.

O conhecimento metacognitivo, portanto, resulta da combinação destas subcategorias, desenvolvendo-se através da conscientização da maneira como as pessoas, as tarefas e as estratégias interagem e influenciam os resultados das atividades cognitivas (FLAVELL; MILLER; MILLER, 1999; RIBEIRO, 2003).

Junto ao conhecimento metacognitivo há o desenvolvimento de outros dois domínios da metacognição que, apesar de se relacionarem de maneira contínua, são interdependentes: o monitoramento e a autorregulação (FLAVELL; MILLER; MILLER, 1999; FELTES, 2014).

O monitoramento metacognitivo é um processo de observação e reflexão sobre os processos cognitivos envolvidos na tarefa a partir dos objetivos de compreensão e lembranças, sendo considerado um processo bottom-up (FELTES, 2014; KORIAT, 2012). É durante a fase de monitoramento que ocorrem julgamentos prospectivos a respeito do processo, que podem ocorrer antes da retenção da informação ou durante o processo de aprendizagem. Estes julgamentos servem como parâmetros que estimam a realização de uma determinada tarefa, e são chamados de: julgamento de facilidade de aprendizagem (ease-of-learning - EOL), julgamento de aprendizagem (judgements-of-learning - JOL) e julgamento de sentimento de saber (feeling-ofknowing - FOK) (FELTES, 2014; SCHELINE, 2016).

A autorregulação é um processo top-down, que parte das conclusões resultantes do processo de monitoramento para avaliar a execução da tarefa e corrigi-la, quando necessário, controlando as atividades cognitivas através dos processos executivos centrais, afim de avaliar e 
orientar as demais operações cognitivas (RIBEIRO, 2003; FELTES, 2014).

Pode-se dizer, então, que observar e refletir a respeito dos processos cognitivos para a compreensão da atividade é o que se chama de monitoramento, enquanto a autorregulação referese ao planejamento, direcionamento do comportamento e tomada de decisões a partir das conclusões resultantes do monitoramento (FELTES, 2014).

Assim, a metacognição está relacionada com a reflexão, com a avaliação interior a respeito de determinada tarefa e a seleção da melhor maneira de resolvê-la. Estas tarefas podem ser referentes aos objetivos gerais ou específicos e, na maioria das vezes, estes objetivos dependem de processos cognitivos como a compreensão da leitura, a escrita, a comunicação e a compreensão oral, o raciocínio lógico e a solução de problemas (GLASS; HOLYOAK, 1986; FLAVELL; MILLER; MILLER, 1999).

A reflexão envolvida na metacognição potencializa o processo de aprendizagem, visto que conduz a uma melhoria dos próprios processos cognitivos do indivíduo: ao identificar o que se conhece a respeito de determinado assunto e também o que não se conhece, é possível elaborar estratégias que venham a colaborar com uma melhora no desempenho da execução da tarefa (RIBEIRO, 2003).

$\mathrm{O}$ ato de aprender a aprender relaciona-se com a atenção necessária para captar estímulos, formular, estabelecer e projetar estratégias, monitorar o progresso cognitivo durante o processo, examinar a informação disponível, aplicar procedimentos para resolver o problema envolvido na tarefa e verificar a sua adequabilidade (FONSECA, 2015). De uma forma mais simplificada, pode-se dizer que há três momentos importantes que contemplam o que Fonseca (2015), com base em Flavell, Miller e Miller (1999), lista a respeito do processo de aprender a aprender: a) identificar o próprio processo cognitivo; b) desenvolver estratégias para lidar com a tarefa; e c) regular as ações mediante planejamento.

O processo de compreender os mecanismos envolvidos na aprendizagem requer elementos metacognitivos: para aprender a aprender é necessário que, primeiramente, o indivíduo seja capaz de tornar consciente seus processos de pensamento para, então, ser capaz de desenvolver estratégias com a intenção de gerenciar e monitorar estes processos (FLAVELL; MILLER; MILLER, 1999; BEBER; SILVA; BONFIGLIO, 2014).

Ao se pensar na metacognição como um suporte para facilitar a aprendizagem é necessário considerar a importância das estratégias metacognitivas para regular as atividades cognitivas e, desta forma, influenciar diretamente na performance da execução destas tarefas (FLAVELL; MILLER; MILLER, 1999; BEBER; SILVA; BONFIGLIO, 2014).

Assim, as estratégias são elementos essenciais para a metacognição e têm como finalidade trazer à consciência os mecanismos que operam na realização de atividades que envolvem tomada de decisões, julgamentos e também em inferências, tornando reconhecíveis as ações que podem ser tomadas para um determinado objetivo cognitivo (STEDILE, 2002; FELTES, 2014).

As estratégias metacognitivas atuam de forma a acompanhar de maneira consciente a realização de tarefas cognitivas. Assim sendo, quanto mais conhecimento o indivíduo possuir sobre suas especialidades, capacidades e limitações, mais clara será a sua decisão a respeito da melhor estratégia a ser utilizada para determinada atividade, podendo permutá-la e modificá-la de acordo com a situação do contexto (BEBBER; SILVA; BONFIGLIO, 2014; FELTES, 2014; STEDILE, 2002). 
O resultado de valorizar a autoanálise e a execução de uma avaliação autônoma reflete na potencialização da aprendizagem a partir da valorização do ato de pensar criticamente, de maneira criativa e divergente diante do apanhado de informações que contextualizam a atividade a ser realizada (PORTILHO, 2006). As estratégias, portanto, surgem a partir da autoavaliação, da possibilidade do sujeito ser capaz de ações de regulação que partem do seu conhecimento a respeito de seu repertório de saberes. Elas exigem que o indivíduo não mais automatize tarefas cognitivas, mas que torne consciente as etapas do processo de execução.

Ao considerar a reflexão e a tomada de consciência como características de aprendizagem é possível identificar grandes grupos de estratégias metacognitivas que são utilizadas, neste trabalho, a partir da perspectiva de Flavell, Miller e Miller (1999), Mayor, Suengas e Marqués (1995 apud PORTILHO, 2006) e Oxford (1990), simplificadas da seguinte forma:

a) gerenciamento de informações: reflexão a respeito do quanto se sabe sobre determinada tarefa, com a associação de um novo conhecimento a um conhecimento prévio, com a ate nção e a alocação dos recursos cognitivos para a realização da tarefa;

b) estratégias de planejamento: regulação do controle cognitivo, com identificação de objetivos e metas, análise, síntese, feedback e adaptação;

c) estratégias de autorregulação e controle: são os elementos do controle, no que diz respeito a capacidade de regulação metacognitiva; capacidades;

d) estratégias de avaliação: encarrega-se do monitoramento e avaliação as próprias

É importante perceber, porém, que a metacognição não é uma construção baseada apenas na reflexão e nas estratégias, mas é um processo complexo, que consiste da relação de fatores internos e externos ao indivíduo, como elementos afetivos, motivacionais e também socioculturais (FELTES, 2014), com os quais o indivíduo se possibilita analisar as exigências da tarefa e as relaciona com a realidade do contexto em questão, de forma a tornar a metacognição capaz de se autoconstruir a partir dos efeitos que causa não apenas no indivíduo, mas também no ambiente (PORTILHO, 2006; STEDILE, 2002).

\section{Método}

A pesquisa realizada neste trabalho tem abordagem qualitativa descritiva, pois permite a descrição das características de um determinado item e sua interpretação, podendo, também, ser utilizada para identificar possíveis relações entre variáveis (GIL, 2010; COSTA, 2011). Este formato de pesquisa permite descrever a ferramenta CCF e analisar suas fases e seus aspectos estruturais, relacionando-os com as estratégias metacognitivas.

Entende-se que para o estudo proposto há a adaptação de instrumentos de análise individuais de estratégias metacognitivas para o coletivo, visto que a ferramenta CCF é utilizada para a tomada de decisões por grupos de atores. Esta adaptação se dá por meio da reformulação do foco das questões que procuram auxiliar a observação de possíveis elementos metacognitivos durante a aplicação da ferramenta CCF.

O objeto de estudo deste trabalho foi a ferramenta CCF, sendo que a linha de pesquisa a ser analisada parte de metodologias aplicadas ao design e desenvolvidas na literatura com base em 
Manzini, Jégou e Meroni (2004), onde se encontra a descrição da mesma.

A coleta de dados deste trabalho se deu de três formas: elaboração de fichas de estrutura, preenchimento de formulário e aplicação de inventário de análise.

Os dois primeiros instrumentos são complementares, sendo que o primeiro procurou identificar e categorizar as fases da ferramenta como itens separados e o segundo visou descrever a composição estrutural da ferramenta e as relações existentes entre suas fases. O terceiro instrumento identificou elementos da metacognição presentes no caso relatado na publicação sobre aplicação da ferramenta CCF.

\subsection{Fichas de estrutura}

A elaboração de fichas de estrutura (APÊNDICE I) deu-se através da decomposição da ferramenta CCF, procurando separar seus elementos estruturais. A ficha de estrutura é um resumo que identifica e caracteriza cada uma das fases para facilitar a análise de seus componentes futuramente, sendo que trabalha com: i. identificação da fase do projeto; ii. descrição da fase do projeto; iii. decomposição da estrutura da fase e a geração de uma lista de verificação a respeito das tarefas que são executadas na fase; iv. criação de palavras-chave relacionadas à fase.

A ficha de estrutura também permitiu separar cada fase da ferramenta CCF em categorias que contextualizam as atividades em quatro grandes grupos relacionados às suas características de execução: Análise (AN) - diz respeito à investigação, exame e reflexão a respeito das informações envolvidas no contexto do projeto; Criatividade (CR) - aplicação das informações através da criação de imagens mentais, sistemas e estruturas que procurem solucionar o problema de projeto; Planejamento $(\mathrm{PL})$ - refere-se à identificação e organização das ações necessárias à solução do projeto; e Síntese (SN) - reunião dos elementos já adquiridos no decorrer do projeto com a intenção de interpretá-los e tomar decisões (MANZINI; JÉGOU; MERONI, 2004).

\subsection{Formulário}

O preenchimento de um formulário (APÊNDICE II) aplicado à ferramenta estudada teve como objetivo identificar os seguintes aspectos: $\mathrm{i}$.organização estrutural da ferramenta e construção das fases; ii. execução das fases durante o processo de uso da ferramenta; iii. representações gráficas; iv relações entre ferramenta e envolvimento dos atores participantes do projeto; v. formas de apresentação dos resultados; vi. análise dos resultados.

O formulário foi dividido a partir de categorias que representam os aspectos acima listados. Para cada uma das categorias foram atribuídas afirmações com a finalidade de investigar se a estrutura da ferramenta CCF permite o desenvolvimento de estratégias metacognitivas durante o seu processo de execução.

O método de respostas fechadas ("concordo" equivalente à 2, "neutro" equivalente à 1 e "discordo" equivalente à 0 ) procurou identificar em quais aspectos a ferramenta possui fraquezas e em quais aspectos possui forças que podem ser utilizadas para relacioná-la com a metacognição.

O escore tabulado a partir das respostas partiu de um número mínimo para que se possa considerar o desenvolvimento de estratégias metacognitivas na ferramenta CCF. Para isso, determinou-se uma afirmação central por categoria que necessitou ter uma pontuação concordante para se chegar a este escore mínimo. Assim, se o escore da aplicação se apresentasse com um valor menor do que 16, significaria que a ferramenta CCF não possui um contexto que forneça os mínimos 
subsídios necessários para o estudo dos elementos que compõem a metacognição.

É importante salientar que por ser um ensaio, este formulário necessita de validação enquanto um instrumento de análise formal.

\subsection{Inventário de análise}

O inventário de análise (APÊNDICE III) foi estruturado a partir dos estudos do referencial teórico abordados anteriormente, sendo que esta análise contemplou a avaliação metacognitiva a partir das estratégias escolhidas para a execução das fases da ferramenta CCF, baseando-se em conhecimento metacognitivo, monitoramento e autorregulação: i.estratégias de planejamento; ii. gerenciamento de informações; iii. estratégias de autorregulação e controle; iv. estratégias de avaliação.

O inventário de análise foi criado a partir de perguntas que procuram investigar os elementos acima citados. As questões com respostas fechadas ("concordo" equivalente à 2, "neutro" equivalente à 1 e "discordo" equivalente à 0 ) procuraram investigaronde os componentes da metacognição são contemplados de forma satisfatória na aplicação da ferramenta CCF, avaliando os dados codificados e organizados em categorias de análise, o que permitiu separar as quatro categorias a serem pesquisadas dentro do caso estudado e atribuir peso = 10 para cada uma.

O inventário de análise foi aplicado a partir do relato de um caso de uso da ferramenta CCF publicado na plataforma Sustainable Everyday Project, o Collaborative Services: social innovation and design for sustainability, organizado por Jégou e Manzini (2008). Esta publicação contém a descrição do uso da ferramenta, contextualizando todo o estudo precedente e os atores envolvidos no seu uso.

Da mesma forma que o formulário, o inventário foi considerado um ensaio, necessitando de validação para sua classificação como instrumento de avaliação de dados.

\section{Apresentação e discussão dos resultados}

Para a realização do trabalho foi necessário identificar as estratégias metacognitivas envolvidas no processo de ensino e aprendizagem em um contexto de projeto. A descrição da ferramenta CCF e a avaliação das fases e seus aspectos estruturais resultaram em um resumo que permitiu separar diferentes categorias de tarefas (Análise, Criatividade, Planejamento, Síntese), de acordo com suas características de execução (APÊNDICE I). Esta análise permitiu identificar os aspectos de resolução de problemas existentes na estrutura da ferramenta CCF e quais os momentos onde há contribuição do conhecimento metacognitivo para a realização das tarefas propostas.

Considerando o resultado dos instrumentos utilizados para este estudo, foi possível relacionar a metacognição com os aspectos presentes na execução das atividades da ferramenta CCF. É importante ressaltar que esta análise partiu de uma avaliação do coletivo, visto que o foco da ferramenta CCF é o uso por grupos de atores. Cabe destacar que a proposta dos instrumentos não passou por processo de validação e, portanto, as considerações feitas estão sujeitas a reavaliação e reconsideração.

Por meio da perspectiva das neurociências, foi possível perceber que a ferramenta CCF permite explorar em maior grau as estratégias relacionadas ao planejamento e ao gerenciamento 
da informação (FLAVELL; MILLER; MILLER, 1999; STEDILE, 2002), estando presentes em diferentes fases da ferramenta CCF e colaborando para a execução das tarefas e envolvimento dos atores.

As estratégias de autorregulação e controle (FLAVELL; MILLER; MILLER, 1999; PORTILHO, 2006; OXFORD, 1990) foram aplicadas com menor eficiência em fases como a de Construção de Cenários. As estratégias relacionadas a avaliação (OXFORD; 1990) também pesaram negativame nte na análise da ferramenta, tornando-se um ponto fraco dentro da estrutura de aplicação da mesma, não apenas nas fases finais da ferramenta, mas também nas fases de análise (APÊNDICE II ; APÊNDICE III).

O cruzamento dos dados feitos a partir dos resultados de cada instrumento permitiu elaborar a relação entre estratégias metacognitivas e aspectos da ferramenta CCF, sendo que:

a) mesmo que de maneira coletiva, a aplicação da ferramenta CCF permite exercitar a reflexão acerca das informações que são coletadas e a procura da melhor maneira de processá-las durante a realização das tarefas propostas em cada fase, o que vem de encontro com os conceitos dos conhecimentos metacognitivos a respeito de tarefas (FLAVELL; MILLER; MILLER, 1990; FELTES, 2014). Esta reflexão que resulta na decomposição da informação serve para tornar os dados coletados aplicáveis no contexto de projeto, procurando facilitar a tomada de decisão evitando erros de julgamento, escassez de informação e até mesmo procurando formas de tornar estas informações mais familiares;

b) as maiores dificuldades encontradas na ferramenta CCF estão relacionadas com a síntese do projeto, nas fases de Construção de Cenários e Representações, sendo que nestas fases concentra-se a categoria de avaliação dos resultados (identificada por meio do formulário) e as estratégias de avaliação (identificadas por meio do inventário). A dificuldade em ser capaz de avaliar e acompanhar de maneira consciente a realização de tarefas metacognitivas é recorrente, tanto no uso de estratégias metacognitivas (PORTILHO, 2006) quanto em resolução de problemas (CLEMENT; TERRAZZAN, 2011; GLASS; HOLYAOK, 1986);

c) viu-se a necessidade de existir dentro da ferramenta meios mais eficazes para avaliar os resultados obtidos, que sirvam como um momento de reflexão coletiva e de regulação em busca de identificar erros recorrentes na execução das tarefas e a melhor forma de saná-los, o que acontece quando há desenvolvimento da metacognição, mesmo que seja em nível individual (OXFORD, 1990; FLAVELL; MILLER; MILLER, 1999);

d) as representações gráficas são elementos importantes para a interação entre os atores e podem servir como um apoio para desenvolver a autorregulação e o controle, uma vez que podem servir como um subsídio para a revisão das tarefas e um ajuste de metas durante a execução das mesmas (FELTES, 2014);

e) a organização das tarefas dentro de um espaço temporal é um elemento importante da metacognição (OXFORD, 1990), e é um quesito não contemplado diretamente no contexto da ferramenta CCF. Percebeu-se que o planejamento das atividades estipuladas através de um cronograma poderia facilitar a execução das tarefas programadas em cada fase, mas que este planejamento deve estar previsto dentro da própria estrutura da ferramenta CCF;

f) há falhas na autorregulação das tarefas dentro das fases da CCF, o que gera dificuldades em realizar ajustes durante a própria execução das atividades. Este ponto aparece como um déficit ao se relacionar com a metacognição, visto que estas correções fazem parte do crescimento 
cognitivo dos indivíduos (PORTILHO, 2006).

De um modo geral, o que se percebe é que, em nível coletivo, a ferramenta demonstra possibilidades de desenvolvimento metacognitivo. Os pontos falhos da ferramenta neste quesito representam uma possibilidade de aperfeiçoa-la para explorar a metacognição enquanto um domínio individual.

Quando fala-se em desenvolvimento da metacognição é importante ter em mente que isto envolve diferentes níveis de consciência, que vão do aprimoramento do pensamento reflexivo e a forma de acessar esta reflexão de maneira consciente até a maneira como expressar o produto destes pensamentos de forma verbal ou não verbal (FELTES, 2014), e que isto pode ocorrer de forma espontânea ou conduzida (PORTILHO, 2006; FELTES, 2014). Desta forma, em ambientes profissionais e educacionais, a ferramenta CCF pode serutilizada como um meio para conduzir estes processos, servindo como um suporte para que os atores envolvidos no projeto experimentem o desenvolvimento dos processos metacognitivos juntamente com os processos cognitivos.

\section{Considerações finais}

A ferramenta CCF é um instrumento importante no contexto de projetos e proporciona aprendizagem colaborativa. Com este trabalho, percebeu-se que a introdução de elementos da metacognição em sua estrutura tem a capacidade de potencializar o uso da ferramenta CCF para a aprendizagem nos grupos de atores.

Como sugestão para trabalhos futuros há a proposta de um estudo para a validação dos instrumentos utilizados para análise dos da dos, o formulário e o inventário, através da comparação de uso destes instrumentos por especialistas em metacognição e especialistas no uso da ferramenta CCF para averiguar se os mesmos podem ser utilizados para a análise da metacognição em diferentes ferramentas e métodos aplicados por grupos que possuam o projeto como principal atividade.

\section{Referências}

AMER, Muhammad; DAIM, Tugrul U; JETTER, Antonie. A review of scenario planning. Futures, v. 46, p. 23-40, 2013.

BEBER, Bernardétte; SILVA, Eduardo da; BONFIGLIO, Simoni Urnau. Metacognição como processo da aprendizagem. Psicopedagogia, v. 31, p. 144-151, 2014.

CATANIA; A. Charles. Aprendizagem: comportamento, linguagem e cognição. Porto Alegre: Artes Médicas Sul, 1999.

CLEMENT, Luiz; TERRAZZAN, Eduardo Adolfo. Educación. Revista Electrónica de Investigación en educación en ciencias, v. 6, p. 87-101, 2011.

COSTA; Marco Antonio F. da. Projeto de pesquisa: entenda e faça. Petrópolis, RJ: Vozes, 2011.

DESIGN FOR SUSTAINABILITY (D4S). Design Orienting Scenario. Disponível em http://www.d4ssbs.org. Acessado em 23 de agosto de 2016.

FELTES, Heloísa Pedroso de Moraes. Cognição e Metacognição: aplicação em uma atividade 
psicolinguística com Teste Cloze. IN: PELOSI, Ana Cristina; FELTES, Heloísa Pedroso de Moraes; FARIAS, Emilia Maria Peixoto (org.). Cognição e linguística: explorando territórios, mapeamentos e percursos. Caxias do Sul, RS: Educs, 2014.

FLAVELL, John H.; MILLER, Patrícia H; MILLER, Scott A. Desenvolvimento cognitivo. Porto Alegre: ArtMed, 1999.

FONSECA, Vitor da. Cognição, neuropsicologia e aprendizagem: abordagem neuropsicológica e psicopedagógica. Petrópolis, RJ: Vozes, 2015.

GIL, Antonio Carlos. Como elaborar projetos de pesquisa. São Paulo: Atlas, 2010.

GLASS, Arnold Lewis; HOLYOAK, Keith James. Cognition. New York: Random House, 1986.

HARTMANN, Patricia; FRANZATO, Carlo; SCALETSKY, Celso Carnos; REYES, Paulo. A representação dos cenários que orientam o processo de projeto. In: 10 CONGRESSO BRASILEIRO DE PESQUISA E DESENVOLVIMENTO EM DESIGN, 2012, São Luís. Anais do 10 Congresso Brasileiro de Pesquisa e Desenvolvimento em Design: 10 -13 de Outubro de 2012.São Luís: EDUFMA, 2012.

JÉGOU, François; MANZINI, Ezio. Collaborative services: social innovation and design for sustainability. Milão: Edizioni POLI.design, 2008. Disponível em http://www.sustainable-everydayproject.net/blog/library-collaborative-services. Acessado em 29 de dezembro de 2016.

KORIAT; Asher. The relationships between monitoring, regulation and performance. Learning and Instruction, v. 22, p. 296-298, 2012.

MANZINI, Ezio; JÉGOU, François; MERONI, Ana. Module B: Design-Oriented Scenarios. In: MARCEL, C; DIEHL, C; RYAN, C. Design for Sustantainability: a step by step aproach. United Nations Environment Programme (UNEP) and Delf University of Technology. Delf: 2004. Disponível em http://www.d4s-sbs.org. Acessado em 23 de agosto de 2016.

MERONI, Ana. Creative communities: people inventing sustainable ways of living. Milão: Edizioni POLI.design, 2007. Disponível em http://www.sustainable-everyday-project.net/blog/librarycreative-communities. Acessado em 29 de dezembro de 2016.

METCALFE, Janet. Metacognitive Process. IN: BJORK, Elizabeth Ligon; BJORK, Robert A. (org). Memory. Califórnia: Academic Press, 1996.

OXFORD, Rebecca. Language Learning Strategies: what every teacher should know. Nova York: Newbury House Publishers, 1990.

PORTILHO, Evelise Maria Labatut. As estratégias metacognitivas de quem aprende e de quem ensina. IN: MALUF, Maria Irene (org). Aprendizagem: tramas do conhecimento, do saber e da subjetividade. Petrópolis: Vozes, 2006.

RIBEIRO, Célia. Metacognição: um apoio ao processo de aprendizagem. Psicologia: Reflexão e Crítica, v. 16, p. 109-116, 2003.

SCHELINI, Patrícia Waltz; et al. Avaliação do monitoramento metacognitivo: análise da produção científica. Aval. psicol., Itatiba, v. 15, p. 57-65, 2016.

STEDILE, Nilva Lúcia Rech. Intervenção metacognitiva como estratégia de aprendizagem em prevenção de problemas de saúde. 2002. 223f. Tese (Doutorado em Enfermagem) - Universidade Federal de São Paulo, Programa de Doutorado em Enfermagem, 2002. 
SUSTAINABLE EVERYDAY PROJECT. SEP Library. Disponível em http://www.sustainable-everydayproject.net. Acessado em 25 de agosto de 2016.

VAN DER HEIJDEN, Kees. Planejamento por cenários: a arte da conversação estratégica. Porto Alegre: Bookman, 2009.

\section{Apêndices}

Apêndice I - Fichas de estrutura

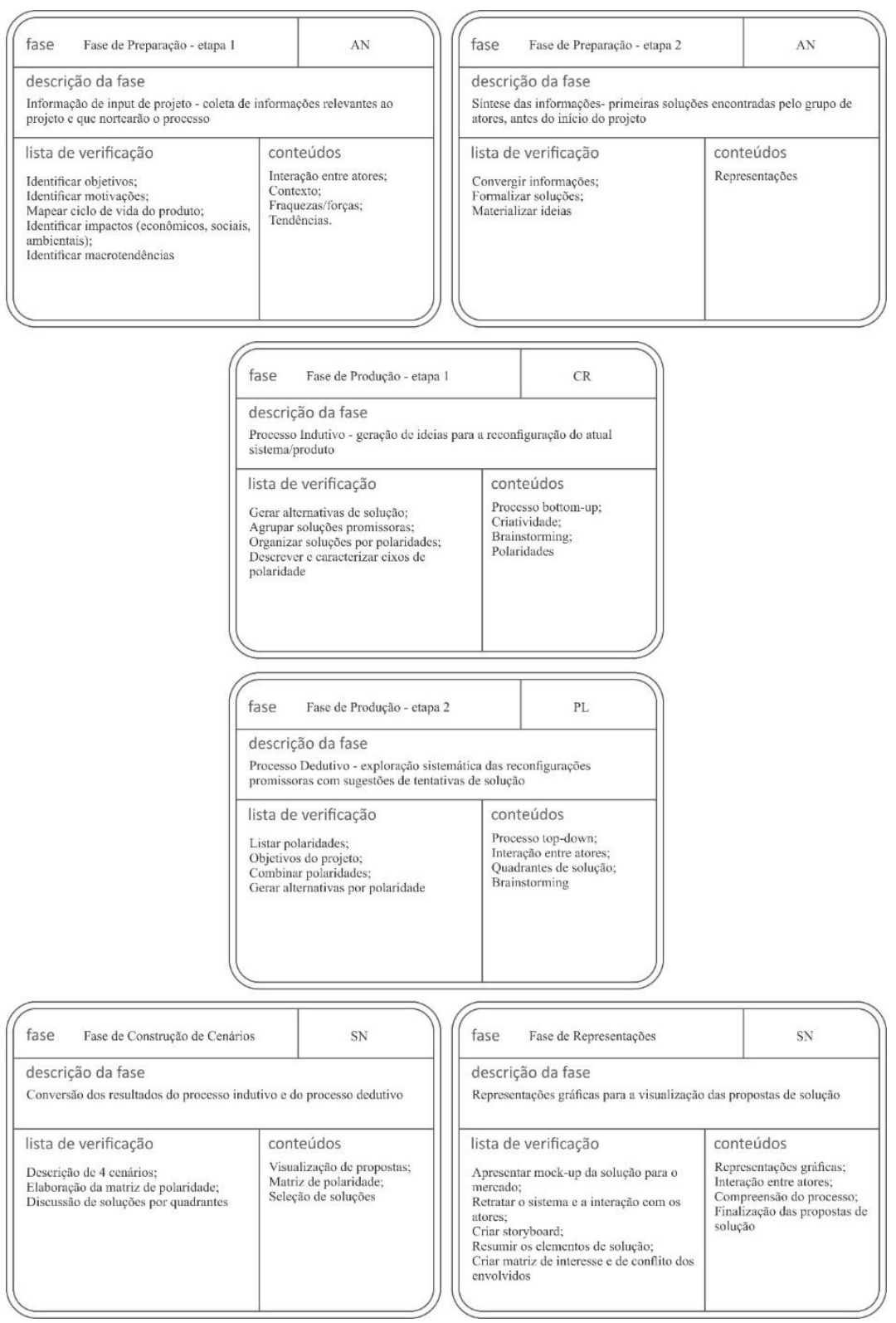




\section{Apêndice II - Formulário}

\begin{tabular}{|c|c|c|c|c|}
\hline \multicolumn{5}{|c|}{ FORMULÁRIO } \\
\hline Categoria & Questão & \multicolumn{2}{|c|}{ Resposta } & Escore \\
\hline \multirow{2}{*}{ Organização estrutural } & É possivel identificar facilmente as caracteristicas de cada fase da CCF & Discordo & - & 0 \\
\hline & A evolução do processo é facilmente identificada em cada fase & Discordo & $\nabla$ & 0 \\
\hline \multirow{2}{*}{ Construção das etapas } & Existe relação clara entre cada fase da CCF & Discordo & - & 0 \\
\hline & Os conceitos centrais de cada fase da CCF são facilmente identificados & Concordo & $\nabla$ & 2 \\
\hline \multirow[t]{2}{*}{ Execução das etapas } & A ferramenta possui subsídios suficientes para a execução de cada fase & Concordo & $\nabla$ & 2 \\
\hline & Há um processo de reflexão-ação para a realização das fases da CCF & Concordo & $\nabla$ & 2 \\
\hline \multirow{3}{*}{ Representação gráfica } & As representações gráficas são importantes para a aplicação da CCF & Concordo & $\nabla$ & 2 \\
\hline & Os dados são facilmente representados através da ferramenta & Discordo & - & 0 \\
\hline & A qualidade do conteúdo das representações gráficas interfere nos resultados da CCF & Concordo & - & 2 \\
\hline \multirow{2}{*}{ Ferramenta $\mathrm{x}$ envolvimento atores } & A ferramenta permite o desenvolvimento individual dos atores & Neutro & - & 1 \\
\hline & A ferramenta permite rotatividade de atores sem interferir no processo & Concordo & - & 2 \\
\hline \multirow{3}{*}{ Apresentação dos resultados } & A a presentação dos resultados é importante para as fases futuras & Concordo & - & 2 \\
\hline & Os resultados são apresentados de forma adequada em cada fase & Discordo & - & 0 \\
\hline & Os resul tados são satisfatoriamente apresentados ao fim do uso da CCF & Discordo & - & 0 \\
\hline \multirow{4}{*}{ Análise dos resultados } & Há subsídio na própria ferramenta para a análise dos resultados & Discordo & - & 0 \\
\hline & Cada fase possui elementos próprios para sua análise & Concordo & - & 2 \\
\hline & Há adequação da fase de representação como final ização das propostas & Discordo & $\nabla$ & 0 \\
\hline & Há facilidade em analisar a proposta final da CCF & Discordo & $\nabla$ & 0 \\
\hline & & \multicolumn{2}{|c|}{ TOTAL } & 23 \\
\hline
\end{tabular}




\section{Apêndice III - Inventário de Análise}

INVENTÁRIO DE ANÁLISE

\begin{tabular}{|c|c|c|c|c|}
\hline Categoria & Questão & Peso & $\begin{array}{l}\text { Discordo (0), Neutro } \\
\text { (1) e Concordo (2) }\end{array}$ & Escore \\
\hline \multirow{7}{*}{$\begin{array}{l}\text { Estratégias de } \\
\text { planejamento }\end{array}$} & Existe controle do andamento das atividades com a finalidade de ter tempo suficiente para cada etapa?* & 1,43 & 0 & \multirow{7}{*}{6} \\
\hline & Os objetivos de cada fase são claros suficientes para todos os atores envolvidos? & 1,43 & 2 & \\
\hline & As estratégias utilizadas durante a execução das fases da ferramenta possuem propósito específico? & 1,43 & 2 & \\
\hline & $\begin{array}{l}\text { Existe um momento de reflexão a respeito do nível do conhecimento antes de se iniciar cada fase da } \\
\text { ferramenta? }\end{array}$ & 1,43 & 1 & \\
\hline & Há espaço para a reflexão sobre diferentes maneiras de resolver o problema apresentado em casa fase? & 1,43 & 2 & \\
\hline & A ferramenta permite escolher a melhor maneira de proceder em cada fase? & 1,43 & 2 & \\
\hline & $\begin{array}{l}\text { A ferramenta permite modificar a qualquer momento aspectos que tenham sido identificados como não } \\
\text { adequados? }\end{array}$ & 1,43 & 0 & \\
\hline \multirow{9}{*}{$\begin{array}{l}\text { Gerenciamento da } \\
\text { informação }\end{array}$} & Há diminuição do ritmo da execução das tarefas quando informações importantes são encontradas? & 1,11 & 2 & \multirow{9}{*}{7,8} \\
\hline & Estas informações recebem a devida atenção por parte dos atores envolvidos durante a tarefa? & 1,11 & 0 & \\
\hline & Novas informações são tratadas com importância? & 1,11 & 1 & \\
\hline & O significado de novas informações é utilizado no processo de execução das tarefas? & 1,11 & 2 & \\
\hline & São criados exemplos próprios a fim de tornar as informações mais significativas? & 1,11 & 2 & \\
\hline & Imagens e diagramas são utilizadas além da etapa de representação gráfica? & 1,11 & 2 & \\
\hline & Novas informações são incorporadas no contexto de cada projeto? & 1,11 & 1 & \\
\hline & $\begin{array}{l}\text { Há es paço para a reflexão e comparação a res peito de novos conhecimentos que possam estar presentes } \\
\text { no repertório do projeto? }\end{array}$ & 1,11 & 2 & \\
\hline & É possível entender o significado geral como sendo maior do que o de cada fase em separada? & 1,11 & 2 & \\
\hline \multirow{8}{*}{$\begin{array}{l}\text { Estratégia de } \\
\text { autorregulação e } \\
\text { controle }\end{array}$} & Os objetivos são revistos durante a execução da ferramenta? & 1,25 & 2 & \multirow{8}{*}{5} \\
\hline & Antes de escolher uma solução, alternativas são consideradas? & 1,25 & 2 & \\
\hline & As alternativas de solução são esgotadas antes de resolver o problema? & 1,25 & 2 & \\
\hline & Etapas anteriores são revisadas com o intuito de entender relações importantes?* & 1,25 & 1 & \\
\hline & Há questionamento a respeito da melhor estratégia a ser utilizada para resolver o problema da etapa? & 1,25 & 0 & \\
\hline & Existem paradas onde é checado o nível de conhecimento dos atores com relação ao projeto em geral? & 1,25 & 0 & \\
\hline & Há momento para a análise do quanto se aprendeu durante a execução das tarefas? & 1,25 & 0 & \\
\hline & $\begin{array}{l}\text { Há momentos para autoquestionamento dos atores com relação ao uso correto das estratégias } \\
\text { empregadas em cada etapa? }\end{array}$ & 1,25 & 1 & \\
\hline \multirow{7}{*}{$\begin{array}{l}\text { Estratégia de } \\
\text { avaliação }\end{array}$} & A ferramenta permite avaliar o desempenho individual dos atores durante a execução das atividades? & 1,43 & 0 & \multirow{7}{*}{5} \\
\hline & É possível avaliar o desempenho coletivo atingido em cada fase da ferramenta? & 1,43 & 2 & \\
\hline & $\begin{array}{l}\text { Existe algum momento onde é possivel questionar se as maneiras encontradas para resolver o problema } \\
\text { são as mais fáceis? }\end{array}$ & 1,43 & 2 & \\
\hline & É possível resumir o que foi aprendido durante o processo de execução das tarefas? & 1,43 & 2 & \\
\hline & A maneira como os objetivos são alcançados é considerada na fase de Repres entações? & 1,43 & 0 & \\
\hline & É possivel avaliar erros cometidos durante o processo de execução de cada tarefa? & 1,43 & 1 & \\
\hline & A ferramenta permite avaliar se os resultados obtidos são satisfatórios? & 1,43 & 0 & \\
\hline
\end{tabular}

